\title{
A REMARK ON THE CONFORMAL CAPACITY OF GRÖTZSCH'S CONDENSER IN SPACE
}

\section{PETER LINDQVIST}

\section{Grötzsch's condenser}

Grötzsch's condenser is a well-known extremal condenser in the complex plane and the corresponding configuration in space has certain applications e.g. for the theory of quasiconformal mappings.

By the conformal capacity of Grötzsch's condenser $\left(B^{n}, J^{n}(r)\right), B^{n}=\left\{x \in \boldsymbol{R}^{n} \mid\right.$ $|x|<1\}$ and $J^{n}(r)=\left\{\left(0, \ldots, 0, x_{n}\right) \mid 0 \leqq x_{n} \leqq r\right\}$, we mean the quantity

$$
v_{n}(r)=\inf _{\varphi} \int_{B^{n}}|\nabla \varphi|^{n} d m \quad(0<r<1) .
$$

Here the infimum is taken among all $\varphi \in C^{1}\left(B^{n}\right)$ with boundary values $\varphi \mid \partial B^{n}=0$ and $\varphi \mid J^{n}(r)=1$. A basic fact is that the integral $\int|\nabla \varphi|^{n} d m$ is conformally invariant. The function $\varphi$ for which (1.1) is actually obtained is not known in space. Nevertheless, good approximations for $v_{n}(r)$ have been obtained.

Replacing $J^{n}(r)$ by a ball with $J^{n}(r)$ as a diameter one obtains the bound

$$
v_{n}(r)<\omega_{n-1}(\overline{\operatorname{ar}} \cosh (1 / r))^{1-n} \quad(0<r<1),
$$

which is equal to the conformal capacity of the enlarged condenser $\left(B^{n}, B^{n}((0, \ldots\right.$ $\ldots, 0, r / 2), r / 2)$ ). Here $\omega_{n-1}$ is the area of the unit sphere in $\boldsymbol{R}^{n}$. However, (1.2) is accurate only for small values of $r$.

A lower bound of the form

$$
v_{n}(r)>A_{n} \log \frac{1+r}{1-r} \quad(0<r<1)
$$

is easily derived via a Möbius transformation that maps $B^{n}$ onto the upper half space in $\boldsymbol{R}^{n}$, where the oscillation lemma of Gehring yields the desired result. See [3] and $[6]$.

A difficult question has been how to achieve natural upper bounds for $v_{n}(r)$ as $r \rightarrow 1-0$. An estimate was given in 1974 by G. Andersson, who calculated that

$$
v_{n}(r)<A_{n} \log \frac{1+r}{1-r}+C_{n} \quad(0<r<1) .
$$


Here $A_{n}$ is the same positive constant as in (1.3) and hence (1.4) is, indeed, relevant for $r$ close to 1. See [2, Theorem 2, Theorem 3, Corollary 1] and [5, Lemma 1].

Andersson's method is based on the fact that in plane the infimum (1.1) is obtained for a well-known function $\varphi(z)$, described by the aid of Jacobi's elliptic sine function. Then one may say $\varphi(z)$ is rotated to give a function in space, admissible for the infimum in (1.1). The objective of our note is to achieve (1.4) more adequately. We are guided by an explicit expression for the $n$-harmonic measure of a diagonal in a ball; c.f. [4].*

\section{An estimate}

The following result, due to Andersson, will be proved by elementary calculus.

Theorem. For $0<r<1$ the estimate

$$
v_{n}(r)<A_{n} \log \frac{1+r}{1-r}+C_{n}
$$

holds. Here $C_{n}$ depends only on $n$ and $A_{n}=\omega_{n-2} / x_{n}^{n-1}, \omega_{n-2}$ being the area of the unit sphere in $\mathbb{R}^{n-1}$ and

$$
\chi_{n}=\int_{0}^{\pi / 2}(\sin t)^{(2-n) /(n-1)} d t .
$$

Proof. (We do not care about the best possible $C_{n}$ but $A_{n}$ is important in view of (1.3).)

Since the integral $\int|\nabla \varphi|^{n} d m$ is conformally invariant, i.e., it is preserved by Möbius transformations, we can carry over the problem to a situation in the upper half-space $H^{n}=\left\{\left(x_{1}, \ldots, x_{n}\right) \mid x_{n}>0\right\}$. To begin with we note that

$$
v_{n}(r)=\mu_{n}\left(\frac{1-\left(1-r^{2}\right)^{1 / 2}}{r}\right),
$$

where $\mu_{n}\left(r^{\prime}\right)$ is the conformal capacity of the condenser $\left(B^{n}, I^{n}\left(r^{\prime}\right)\right), I^{n}\left(r^{\prime}\right)=$ $\left\{\left(0, \ldots, 0, x_{n}\right) \mid-r^{\prime} \leqq x_{n} \leqq r^{\prime}\right\}$. Thus we have to estimate $\mu_{n}(r)$. For a fixed $r$, $0<r<1$, there is a Möbius transformation $\gamma$ from $B^{n}$ onto $H^{n}$ such that

$$
\gamma(0, \ldots, 0, \pm r)=\left(0, \ldots, 0,2 \frac{1 \mp r}{1 \pm r}\right)
$$

and $\gamma\left(I^{n}(r)\right)$ is a certain line segment. If $\varphi$ is admissible for the auxiliary condenser $\left(B^{n}, I^{n}(r)\right)$, then

$$
\int_{B^{n}}|\nabla \varphi|^{n} d m=\int_{H^{n}}\left|\nabla\left(\varphi \circ \gamma^{-1}\right)\right|^{n} d m
$$

by conformal invariance.

* A general idea of the principles involved is given in Granlund, S. Lindqvist, P., and Martio, O.: F-harmonic measure in space, Ann. Acad. Sci. Fenn. Ser. A. I. Math. 7 (1982), $233-247$. 
Let $\theta$ denote the angle between the positive $x_{n}$-axis and $x \in \bar{H}^{n}$, i.e.,

for $x \neq 0$. The function

$$
\cos \theta=x_{n} /|x|, \quad 0 \leqq \theta \leqq \pi / 2
$$

$$
V(\theta)=\frac{1}{x_{n}} \int_{\theta}^{\pi / 2}(\sin t)^{(2-n) /(n-1)} d t \quad(0 \leqq \theta \leqq \pi / 2)
$$

is the key to (2.1). Our explanation is that $V$ is in fact the so-called $n$-harmonic measure of $X=\left\{\left(0, \ldots, 0, x_{n}\right) \mid x_{n} \geqq 0\right\}$ taken with respect to the domain $H^{n} \backslash X$, and hence $V$ is somehow close to "the right function". (Especially, $V$ is a solution of the $n$-harmonic equation $\operatorname{div}\left(|\nabla V|^{n-2} \nabla V\right)=0$ in $H^{n} \backslash X$.) See [4].

A simple calculation in spherical coordinates gives

$$
\int_{\substack{r_{1}<|x|<r_{2} \\ x_{n}>0}}|\nabla V|^{n} d m=\frac{\omega_{n-2}}{x_{n}^{n-1}} \log \frac{r_{2}}{r_{1}},
$$

where we are interested in the choices

$$
r_{1}=2 \frac{1-r}{1+r}, \quad r_{2}=2 \frac{1+r}{1-r} .
$$

Unfortunately, the integral $\int|\nabla V|^{n} d m$ is infinite when taken over $H^{n}$. Therefore we are forced to adjust $V$ near the points 0 and $\infty$. To this end, define

$$
\zeta(x)=\left\{\begin{array}{l}
0 \text { if }|x| \geqq 2 r_{2} \text { or }|x| \leqq r_{1} / 2 \\
1 \text { if } r_{1} \leqq|x| \leqq r_{2} \\
\log \left(2|x| / r_{1}\right) / \log 2 \text { if } r_{1} / 2 \leqq|x| \leqq r_{1} \\
\log \left(|x| / 2 r_{2}\right) / \log (1 / 2) \text { if } r_{2} \leqq|x| \leqq 2 r_{2}
\end{array}\right.
$$

for $x \in H^{n}$ and consider the admissible function $\zeta V$.

By (2.4)

$$
\mu_{n}(r) \leqq \int_{H^{n}}|\nabla(\zeta V)|^{n} d m
$$

By virtue of (2.5) we obtain

$$
\mu_{n}(r) \leqq \frac{\omega_{n-2}}{x_{n}^{n-1}} \log \left(\frac{1+r}{1-r}\right)^{2}+\int_{0<\zeta<1}|\nabla(\zeta V)|^{n} d m
$$

and a rough upper bound for the last integral is

$$
2^{n-1}\left\{\int_{0<\zeta<1}|\nabla V|^{n} d m+\int_{0<\zeta<1}|\nabla \zeta|^{n} d m\right\} \leqq 2^{n}\left\{\frac{\omega_{n-2} \log 2}{x_{n}^{n}-1}+\frac{\omega_{n-1}}{(\log 2)^{n-1}}\right\}=C_{n} .
$$

Thus we have

$$
\mu_{n}(r) \leqq 2 A_{n} \log \frac{1+r}{1--r}+C_{n},
$$

and a substitution in (2.3) gives (2.1). This concludes our proof. 


\section{References}

[1] Ahlfors, L.: Möbius transformations in several dimensions. - Ordway professorship lectures in mathematics, University of Minnesota, 1981.

[2] Andersson, G.: Extremal rings in $n$-space for fixed and varying $n$. - Ann. Acad. Sci. Fenn. Ser. A. I. Math. $575,1974,1-21$.

[3] Gehring, F.: Rings and quasiconformal mappings in space. - Trans. Amer. Math. Soc. 101, $1961,499-519$.

[4] Lindevist, P.: The differential equation $\operatorname{div}\left(|\nabla u|^{n-2} \nabla u\right)=0$ in $n$-dimensional space. - ReportHTKK-Mat-A182, 1981, 1-14.

[5] Miniowitz, R.: Distortion theorems for quasiregular mappings. - Ann. Acad. Sci. Fenn. Ser. A. I. Math. 4, 1978/1979, 63-74.

[6] Mostow, G.: Quasi-conformal mappings in $n$-space and the rigidity of hyperbolic space forms. Inst. Hautes Études Sci. Publ. Math. 34, 1968, 53-104.

Helsinki University of Technology

Institute of Mathematics

SF-02150 Espoo 15

Finland

Received 18 January 1984 